\title{
Bilateral subcutaneous pyelovesical bypass in a Hautmann neobladder followed by a mononeuropathy multiplex and an underlying polyarteritis nodosa diagnosis
}

\author{
Konstantina G. Yiannopoulou ${ }^{1}$, Aikaterini I. Anastasiou ${ }^{2}$, Ioannis Katafigiotis ${ }^{2}$, \\ Dimitrios Papadopoulos ${ }^{3}$, Ioannis Anastasiou ${ }^{2}$ \\ ${ }^{1}$ Neurology Department, Henry Dunant Hospital Center, Athens, Greece; \\ $21^{\text {st }}$ University Urology Clinic, Laiko Hospital, Athens Greece; \\ ${ }^{3}$ Athens Medical Centre-Paleo Phaliro Clinic, Athens, Greece.
}

\begin{abstract}
Summary Subcutaneous pyelovesical bypasses are the best choice for the long-term palliative treatment of ureteral obstructions. In rare cases this obstruction is due to polyarteritis nodosa. We present the only reported patient with a bilateral Detour bypass in a Hautmann's neobladder. The patient also suffers from polyarteritis nodosa.
\end{abstract}

KEY WORDS: Bilateral; Detour bypass; Hautmann neobladder; polyarteritis nodosa.

Submitted 10 August 2019; Accepted 1 September 2019

\begin{abstract}
INTRODUCTION
Subcutaneous pyelovesical bypasses or Detour bypass is the safest and most effective method for the long-term palliative treatment of ureteral obstructions (1). Ureteral obstruction can be caused from either benign or malignant disease (1). We present a rare case of bilateral subcutaneous pyelovesical bypass in a Hautmann neobladder in a 66-year-old man. To our knowledge this is the first case ever reported. Three months after the second bypass, a mononeuropathy multiplex appeared and polyarteritis nodosa (PON) was diagnosed in the same patient. To our knowledge, five cases of bilateral ureteral stenosis and 16 of unilateral ones due to (PON) have been reported in the literature so far (2). None of them has been occurred in a patient with a neobladder.
\end{abstract}

\section{Case presentation \\ A 66-year-old man was presented to our Urology Department ( $1^{\text {st }}$ University Urology Clinic, Laiko hospital, Athens, Greece), with a bladder carcinoma. We per- formed an endoscopic bladder resection and the final histology revealed a pT2 muscle invasive bladder tumour. A radical cyctectomy was performed with Hautmann neobladder as diversion. Six months later an obstructed uropathy manifested due to left ureter steno- sis and thus we placed a nephrostomy for temporary management. The final treatment was achieved with the placement of a left subcutaneous pyelovesical bypass. Three years later the patient's right ureter presented also stenosis. Initially we placed a double J stent which how-}

ever did not achieve a final solution of the obstruction. Consequently, we performed a second subcutaneous pyelovesical bypass in the right ureter (Figure 1).

Three months later, the patient was admitted with right foot and left wrist drop, fever and myalgias. A neurophysiologic evaluation revealed a mononeuropathy multiplex affecting peroneal and radial nerves with both motor and sensory deficits. A mild proximal muscle weakness and a severe muscle tenderness were present. The diagnosis of PON was based upon muscle biopsy from right quadriceps which revealed typical necrotizing vasculitis in medium size arteries in conjunction with elevated sedimentation rate $(107 \mathrm{~mm} / \mathrm{h})$ and rheumatoid factor $(73 \mathrm{IU} / \mathrm{ml})$. The patient was managed with oral prednisolone $(1 \mathrm{mg} / \mathrm{kg})$, which resulted in gradual resolution of his symptoms.

\section{Discussion}

Bladder cancer is more common in men than in women and usually does not involve the muscle wall. For this reason it is mainly treated by endoscopic transurethral resection of the bladder tumor (TURBT). In our male patient the first surgery was a TURBT, which demonstrated cancer infiltrating the bladder muscle wall. Those patients are at higher risk and therefore are treated with major surgery to remove the bladder.

Subcutaneous pyelovesical bypasses are considered to be the best choice for ureteric obstruction, in comparison to other methods, such as the J stent. It is not associated with septicemia and irritative bladder symptoms or frequent infections (1). The fact that there is no participation of the impaired ureter is the main difference between pyelovesical bypasses and other methods (1). Ureteral obstruction may be caused by benign as well as malignant disease, but is also an important complication of modern surgeries $(1,3)$. In fact, uretero-ileal anastomotic stricture (UIAS) in orthotopic ileal neobladder can develop in $4-10 \%$ of cases during a follow-up time of 6 36 months (3). In our case it was caused by fibrosis in the site of the anastomosis of the ureter with the neobladder. Other methods, which have been used to treat this complication, are percutaneous nephrostomy 
Figure 1.

Placement of the second subcutaneous pyelovesical bypass in the right kidney.

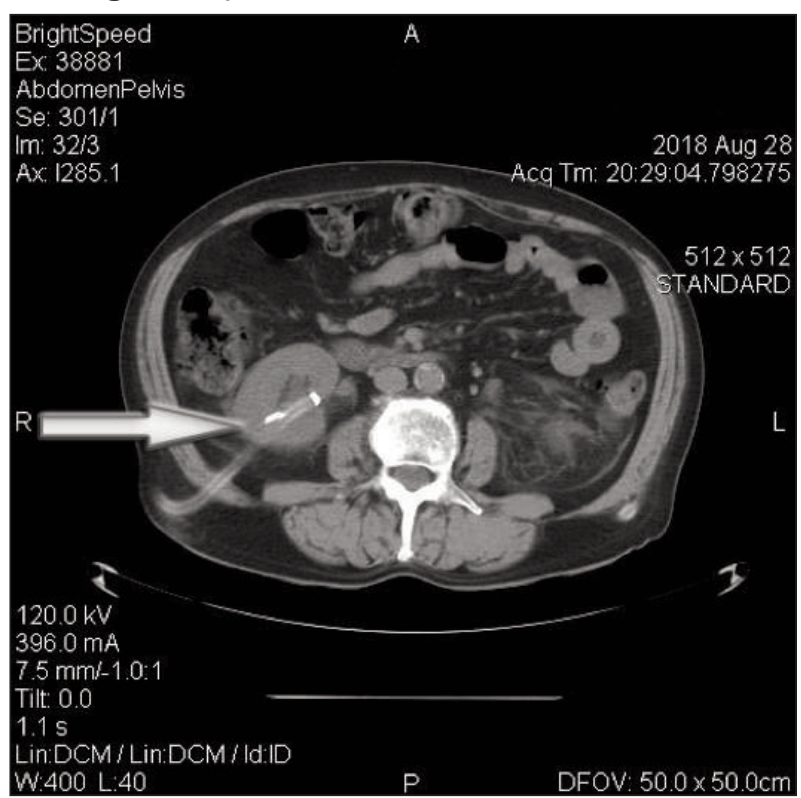

and double J stents. In our patient both methods where applied before the use of subcutaneous pyelovesical bypasses, however their efficiency was poor and the Detour was the final treatment. Nevertheless, this is the first case in the literature where a bilateral ureteric obstruction after Hautmann neobladder was managed succesfully with a bilateral pyelovesical bypass.

Several months after the second Detour procedure, patient presented with multiple mononeuropathies and symptoms of systemic inflammation. He was finally diagnosed with PON, an entity that did not show previous clinical or laboratory signs of its existence in our patient. However, ureteral obstruction has been described as a manifestation of PON (2). It is supposed to result from vasculitis of periureteral vessels. To our knowledge, five cases of bilateral ureteral stenosis and 16 of unilateral ones due to (PON) have been reported in the literature so far (2), two of them as the first manifestation of the disease (4). We suggest that the vasculitic background in our patient due to his underlying polyarteritis pathology in conjunction with the major surgical procedure in his bladder contributed to the unprecedented occurrence of such a severe bilateral ureteral obstruction after Hartmann neobladder surgical construction that only Detour intervention could resolve.

\section{Conclusions}

Although UIAS in orthotopic ileal neobladder may develop in $4-10 \%$ of cases in the follow-up period of 6-36 months, bilateral UIAS can also occur.

Subcutaneous pyelovesical bypasses (Detour bypass) can be applied bilaterally with safety and long-term efficacy. Furthermore, additional underlying causes of the obstructive process, like systematic vasculitis. should be suspected in these extremely rare cases.

\section{REFERENCES}

1. Wrona AJ, Zgajewski J, Kopeć N, Chodor D, Kopcza P, Klekot S. Subcutaneous pyelovesical bypass - Detour bypass - as a solution for ureteric obstruction. Cent European J Urol. 2017; 70:429-433.

2. Bolat D, Zumrutbas AE, Baser A, Tuncay L. Spontaneous ureteral rupture in a patient with polyarteritis nodosa. Int Urol Nephrol. 2016; 48:223-4.

3. Mohamed Wishahi, Hossam Elganzoury, Amr Elkhouly. Detour technique, dipping technique, or ileal bladder flap technique for surgical correction of uretero-ileal anastomotic stricture in orthotopic ileal neobladder. Int Braz J Urol. 2015; 41:796-803.

4. Jois R, Gupta A, Krishnamurthy S. Ureteric vasculitis, an unusual presentation of polyarteritis nodosa: a case report. Int J Rheum Dis. 2015; 18:577-9.

\section{Correspondence}

Konstantina G. Yiannopoulou, MD

k.giannopoulou.14@hotmail.com

Neurology Department, Henry Dunant Hospital Center, Athens (Greece)

Aikaterini I. Anastasiou, MD (Corresponding Author)

ekati2@otenet.gr

Ioannis Katafigiotis, MD

katafigiotis.giannis@gmail.com

Ioannis Anastasiou, MD

aikatianast@gmail.com

$1^{\text {st }}$ University Urology Clinic, Laiko Hospital

Ag. Thoma 17, Athens 11527 (Greece)

Dimitrios Papadopoulos, MD

dimipapuro@yahoo.gr

Athens Medical Centre-Paleo Phaliro Clinic, Athens (Greece) 